OPEN ACCESS

Edited by:

Allison Hansen,

University of California, Riverside,

United States

Reviewed by:

Matthew Doremus,

University of Arizona, United States

Margaret Thairu,

University of Wisconsin-Madison,

United States

*Correspondence:

Shu-Sheng Liu

shshliu@zju.edu.cn

Specialty section:

This article was submitted to

Microbial Symbioses,

a section of the journal

Frontiers in Microbiology

Received: 11 July 2021

Accepted: 31 August 2021

Published: 30 September 2021

Citation:

Shan H-W and Liu S-S (2021) The

Costs and Benefits of Two Secondary

Symbionts in a Whitefly Host Shape

Their Differential Prevalence

in the Field.

Front. Microbiol. 12:739521. doi: 10.3389/fmicb.2021.739521

\section{The Costs and Benefits of Two Secondary Symbionts in a Whitefly Host Shape Their Differential Prevalence in the Field}

\author{
Hong-Wei Shan ${ }^{1,2}$ and Shu-Sheng Liu ${ }^{2 *}$ \\ 1 State Key Laboratory for Managing Biotic and Chemical Threats to the Quality and Safety of Agro-products, Key \\ Laboratory of Biotechnology in Plant Protection of Ministry of Agriculture and Zhejiang Province, Institute of Plant Virology, \\ Ningbo University, Ningbo, China, ${ }^{2}$ Ministry of Agriculture Key Laboratory of Molecular Biology of Crop Pathogens \\ and Insects, Institute of Insect Sciences, Zhejiang University, Hangzhou, China
}

Insects commonly harbor maternally inherited intracellular symbionts in nature, and the microbial partners often exert influence on host reproduction and fitness to promote their prevalence. Here, we investigated composition of symbionts and their biological effects in the invasive Bemisia tabaci MED species of a whitefly complex. Our field surveys revealed that populations of the MED whitefly, in addition to the primary symbiont Portiera, mainly contain two secondary symbionts Hamiltonella, which is nearly fixed in the host populations, and Cardinium with infection frequencies ranging from 0 to $86 \%$. We isolated and established Cardinium-positive and Cardinium-free whitefly lines with a similar nuclear genetic background from a field population, and compared performance of the two whitefly lines. The infection of Cardinium incurred significant fitness costs on the MED whitefly, including reduction of fecundity and egg viability as well as delay in development. We then selectively removed Hamiltonella from the Cardinium-free whitefly line and compared performance of two whitefly lines, one harboring both Portiera and Hamiltonella and the other harboring only Portiera. While depletion of Hamiltonella had little or only marginal effects on the fecundity, developmental rate, and offspring survival, the Hamiltonella-free whitefly line produced very few female offspring, often reducing the progeny female ratio from about $50 \%$ to less than $1 \%$. Our findings indicate that the varying costs and benefits of the association between these two symbionts and the MED whitefly may play an important role in shaping their differential prevalence in the field.

Keywords: whitefly, endosymbionts, vertical transmission, host fitness, sex ratio

\section{INTRODUCTION}

Symbiotic microorganisms are associated with a broad range of invertebrates in nature. The symbionts are generally divided into primary and secondary symbionts based on that whether or not their association is essential for host development and survival (Douglas, 1989; Baumann, 2005). Insects regularly are associated with a variety of symbionts that confer specific benefits to hosts to keep the symbionts being maintained in host populations (Feldhaar, 2011; Douglas, 2015; 
Ayoubi et al., 2020). For example, in some pea aphids, "Candidatus Hamiltonella defensa" (hereafter Hamiltonella) provides protection against parasitoid wasps (Oliver et al., 2003; Oliver and Higashi, 2019). In addition, some symbionts including Wolbachia sp. and "Candidatus Cardinium hertigii" (hereafter Cardinium) manipulate host reproduction leading to variation of sex ratio in various haplodiploid insects and increasing fitness of infected females in diploid insects (ZchoriFein et al., 2004; Werren et al., 2008; Nguyen et al., 2017). These symbionts are maintained through host generations by vertical transmission from parents to offspring and may vary in infection frequencies among host populations, possibly in association with manipulation of host reproduction and/or fitness (Turelli and Hoffmann, 1991; Harris et al., 2010; Himler et al., 2011; McLean et al., 2018).

Bemisia tabaci is a whitefly complex consisting of over 40 cryptic species, some of which are economically important pests such as the two widespread invasive species, tentatively named as Middle East Asia Minor 1 (MEAM1) and Mediterranean (MED) (Liu et al., 2007; De Barro et al., 2011; Hu et al., 2011; Shadmany et al., 2018; Misaka et al., 2020). These phloem feeding insects have a haplodiploid genetic system in which a fertilized egg develops into a diploid female and an unfertilized egg develops into a haploid male (Byrne and Bellows, 1991). The whiteflies harbor the primary symbiont "Candidatus Portiera aleyrodidarum" (hereafter Portiera) and seven known secondary symbionts, including Hamiltonella and Cardinium (Zchori-Fein and Brown, 2002; Weeks et al., 2003; Gottlieb et al., 2008; Bing et al., 2013a,b; Lei et al., 2021). In addition, these symbionts often contain some strains with genetic differentiations (Kanakala and Ghanim, 2019). Portiera, like some other primary symbionts of phloem-feeding insects, provide essential amino acids as well as carotenoids for its whitefly hosts (Sloan and Moran, 2012). The secondary symbionts have diverse effects on their hosts. For example, the Rickettsia confers general benefits to host fitness (Himler et al., 2011) and assists its host in resistance to entomopathogen Pseudomonas syringae in MEAM1 whitefly (Hendry et al., 2014); the Hamiltonella influences host sex ratio via provisioning of nutrients in MEAM1 whitefly (Shan et al., 2019; Wang Y. B. et al., 2020), and some Cardnium and Rickettsia strains confer lower host fitness in B. tabaci MED and SSA1-SG3 species, respectively (Fang et al., 2014; Ghosh et al., 2018; Zhao et al., 2018). In general, whiteflies of this species complex are infected with multiple symbionts and the dynamics of bacterial infections vary among host species/populations (Zchori-Fein et al., 2014). Yet, the factors causing these variations as well as the underlying mechanisms are poorly understood.

This study concerns the natural occurrence of whitefly secondary symbionts and the fitness costs/benefits associated with their infections in the B. tabaci whitefly, focusing on the invasive MED species, which is one group of notorious agricultural pests worldwide having developed high resistance to insecticide (Wang et al., 2020a,b). The MED whiteflies includes four phylogenetic clades Q1, Q2, Q3, and ASL, and Q1 is the most widely distributed genotype in most regions, especially in China (Chu et al., 2008; Gueguen et al., 2010; Gauthier et al., 2014). We surveyed symbionts in different geographical populations of MED in China, and found that the whitefly populations belong to the Q1 clade and were mainly infected with two secondary endosymbionts, Cardinium and Hamiltonella, with varying frequencies of infection among host populations. We then assessed the fitness costs/benefits associated with these two secondary symbionts by manipulating the presence/absence of the symbionts and comparing performance of whitefly populations with or without a given symbiont. Our results indicate that while Cardinium exerts some fitness cost on the host, Hamiltonella plays an essential role for the host to maintain a normal sex ratio, demonstrating diverse functions of different symbionts residing in the same host. We also discuss the association of the different functions of the two symbionts with their varying prevalence in natural host populations.

\section{MATERIALS AND METHODS}

\section{Whitefly Collection and Symbiont Identification}

Field populations of $B$. tabaci were collected in different geographical locations in China from 2012 to 2014 (Supplementary Table 1). Total DNA was extracted from individual insect with Lysis buffer containing Tris-HCl, EDTA Non-idet P-40 and proteinase $\mathrm{K}$ as described previously (Shan et al., 2014). Each individual whitefly was ground in $50 \mu \mathrm{L}$ of ice-cold lysis buffer by Grinding Mill and then incubated at $65^{\circ} \mathrm{C}$ for $2 \mathrm{~h}$ and $100^{\circ} \mathrm{C}$ for $10 \mathrm{~min}$. Extractions were then centrifuged briefly and stored at $20^{\circ} \mathrm{C}$. The species of $\mathrm{B}$. tabaci were identified using mitochondrial cytochrome oxidase $I$ $(m t C O I)$ polymerase chain reaction restriction fragment-length polymorphism (PCR-RFLP) with the enzyme TaqI (Bosco et al., 2006), and further checked with $m t C O I$ sequencing. All the $m t C O I$ sequences generated in this work were deposited in NCBI GenBank.

The whiteflies identified as the MED species were used for detecting symbiont infection. The primary symbiont Portiera was first detected to confirm DNA quality. Then identifications of all seven known secondary symbionts including Hamiltonella, Rickettsia, Cardinium, Wolbachia, Arsenophonus, Fritschea, and Hemipteriphilus in the $B$. tabaci whiteflies were conducted using their specific PCR primers targeting the $16 \mathrm{~S}$ rRNA or 23S rRNA gene and their sequences. The primer sequences of mtCOI and symbionts are listed in Supplementary Table 2. All the $16 S$ rRNA sequences generated in this work were deposited in NCBI GenBank.

\section{Isolation and Establishment of $\mathrm{C}^{+}$and $C^{-}$Whitefly Lines}

The MED population was originally collected from eggplants in Maoming, Guangdong Province, China in 2013 (population no. 11 in Supplementary Table 1), and maintained on cotton plants (Gossypium hirsutum cv. Zhe-Mian 1793) at $26^{\circ} \mathrm{C}$, a photoperiod of 14:10 light/dark (L/D) and 60-80\% relative humidity. The infection of Cardinium was $63.6 \%$ in the original population (Supplementary Table 1). The female lines of 
Cardinium-positive $\left(\mathrm{C}^{+}\right)$and Cardinium-free $\left(\mathrm{C}^{-}\right)$were isolated from the colony after testing by PCR. Briefly, 80 mated females were collected, and each female was reared in isolation on a cotton leaf, enclosed in a leaf-clip cage, to feed and oviposit for 7 days. The females were then collected and examined individually for the presence $\left(\mathrm{C}^{+}\right)$or absence $\left(\mathrm{C}^{-}\right)$of Cardinium. Twenty-five days later, the progenies of $\mathrm{C}^{+}$females and those of $\mathrm{C}^{-}$females were, respectively, pooled together and reared on new cotton plants in two separate insect rearing cages.

In consideration of the possible genetic heterogeneity between the two whitefly lines, we introgressed the $\mathrm{C}^{-}$line into the $\mathrm{C}^{+}$ line over 6 generations to homogenize their nuclear background following the protocol as described by Himler et al. (2011). In every introgression, about $30 \mathrm{C}^{-}$males were mated with about 30 virgin $\mathrm{C}^{+}$females. Subsequently $\mathrm{C}^{-}$males were continuously mated to the introgressed virgin $\mathrm{C}^{+}$female progenies for six consecutive generations. Thereafter, the introgressed $\mathrm{C}^{+}$line and $\mathrm{C}^{-}$line were used for comparing their fitness. We confirmed that the $\mathrm{C}^{+}$line harbor the primary symbiont Portiera, and the two secondary symbionts Hamiltonella and Cardinium, and the $\mathrm{C}^{-}$ line harbor only Portiera and Hamiltonella prior to experiments.

\section{Observing Effects of Cardinium on Host Fitness and Vertical Transmission of the Symbiont}

To examine the effects of Cardinium on host fitness and reproduction, we conducted four treatments of mating between the $\mathrm{C}^{+}$and $\mathrm{C}^{-}$lines including $\mathrm{C}^{+} q \times \mathrm{C}^{+} \sigma^{x}, \mathrm{C}^{-} q \times \mathrm{C}^{-} \sigma^{7}$, $\mathrm{C}^{+} q \times \mathrm{C}^{-} \sigma^{7}$, and $\mathrm{C}^{-} q \times \mathrm{C}^{+} \sigma^{7}$. In each replicate of a given mating treatment, a single virgin female and a single virgin male were reared together on a leaf of a cotton plant, enclosed in a leafclip cage, to feed, mate, and oviposit for 7 days. The adults were then removed and the eggs in each replicate were counted. After a further 10 days, the nymphs in each replicate were counted, and then on the 22nd, 27th, 32nd, and 37rd day since the removal of the females used in the mating treatments, adult progenies were collected, sexed, and counted. From these data, we were able to calculate the number of eggs laid per female, percentage of egg hatching, survival from the 1st instar nymph to adulthood, and number of adult progenies and their sex ratio. To observe the fidelity of vertical transmission of Cardinium, we randomly collected adult progenies from each of the four mating treatments to detect the presence of the bacterium using PCR with its specific primer (Supplementary Table 2).

\section{Antibiotic Treatment to Eliminate Hamiltonella}

To assess effects of the other secondary symbiont Hamiltonella on the insect, we used a cocktail of antibiotics to specifically eliminate the bacterium from the $\mathrm{C}^{-}$whitefly line which harbored only Portiera and Hamiltonella. This cocktail of antibiotics had been used successfully to remove this symbiont from pea aphids and from the MEAM1 species of the B. tabaci whitefly complex (Douglas et al., 2006; Tsuchida et al., 2010; Shan et al., 2019). Hundreds of adults (F0) were fed for 4 days with an artificial diet composed of ampicillin, gentamycin, and cefotaxime (each at $500 \mu \mathrm{g} / \mathrm{ml}$ ) mixed with $25 \%$ sucrose, and the artificial diets were renewed every 2 days. The control sucrose diet solution was supplied without antibiotics. Immediately after the feeding, the whiteflies were transferred to cotton plants to feed and oviposit for 10 days, and then their progenies (F1) were maintained on the plants until they developed to adults in 30 days. To ameliorate the potentially direct effects of antibiotics on the insects, the offspring (F1) adults were used to test the presence of symbionts and conduct bioassays.

\section{Observing Effects of Hamiltonella on Host Reproduction and Vertical Transmission of the Symbiont}

To observe effects of Hamiltonella on the reproduction of MED, we used F1 adults of the Control treatment (CK) where all individuals naturally harbored Hamiltonella, and those of F1 whiteflies of the antibiotic treatment (AT) that were depleted of Hamiltonella to conduct four treatments of mating: $\mathrm{CK} \rho \times \mathrm{CKo}^{\top}$, $\mathrm{AT} \phi \times \mathrm{ATo}^{7}, \mathrm{CK} q \times \mathrm{ATo}^{7}$, and $\mathrm{AT} q \times \mathrm{CKo}^{7}$. In each replicate of a given mating treatment, a single virgin female and a single virgin male were reared together on a leaf of a cotton plant, enclosed in a leaf-clip cage, to feed, mate, and oviposit for 7 days. Then the female and male of the pair were collected and examined separately for the presence/absence of symbionts using qPCR. Adult progenies (F2) of the mating treatments were collected on the 22nd, 27th, 32nd, and 37rd day since the removal of F1 adults, sexed and counted. To observe the vertical transmission of Hamiltonella, we randomly collected some F2 progenies from each of the four mating treatments to detect the bacterium using qPCR, and detection of the Portiera of these whiteflies was also conducted as a reference.

\section{Assessing Effects of Hamiltonella on Host Fitness}

Because F2 progenies produced by F1 whiteflies of the antibiotic treatment (AT) (that were depleted of Hamiltonella) were nearly all males, experiments to examine the effects of Hamiltonella on host fitness were not feasible with F2 whiteflies. We thus assessed the effects of Hamiltonella on host fitness using F1 adults in the following three treatments: (1) Unmated (CK), a single virgin female of the CK whitefly which was supposed to produce only male progenies; (2) CK, a female and a male of the CK whiteflies; (3) AT, a female and a male from the antibiotic treatment. The experimental protocol was the same as that used for observing the effects of Cardinium on host fitness, as described above.

\section{Determining Locations of Symbionts in Whitefly Hosts}

The presence and localization of symbionts in the two whitefly lines were observed using fluorescence in situ hybridization analysis with the protocol of Shan et al. (2014). Insects were fixed in Carnoy's fixative (ethanol/chloroform/glacial acetic acid, 6:3:1) overnight, decolorized in $6 \% \mathrm{H}_{2} \mathrm{O}_{2}$ in ethanol for $2 \mathrm{~h}, 0.1 \%$ Triton $\mathrm{X}-100$ for $1 \mathrm{~h}$ and hybridized overnight in hybridization buffer (20 mmol/lTris- $\mathrm{HCl}(\mathrm{pH} 8.0)$, $0.9 \mathrm{~mol} / \mathrm{l} \mathrm{NaCl}, 0.01 \%$ sodium dodecyl sulfate, and $30 \%$ 
formamide) with $10 \mathrm{pmol}$ of fluorescent probes $/ \mathrm{ml}$. The three symbionts Portiera, Hamiltonella, and Cardinium were detected with their specific probes BTP1-Cy3 (5'-Cy3-TGTCAG TGTCAGCCCAGAAG-3') (Gottlieb et al., 2006), BTH-Cy5 (5'-Cy5-CCAGATTCCCAGACTTTACTCA-3') (Gottlieb et al., 2008), and/or Card-Cy5 (5'-TATCAATTGCAGTTCTAGCG-3') (Matalon et al., 2007), respectively.

\section{Quantitative PCR for Assessing Symbionts Densities}

The quantity of symbionts was assessed using quantitative PCR (qPCR) with the SYBR ${ }^{\circledR}$ Premix Ex Taq ${ }^{\text {TM }}$ (Takara) and BioRad CFX96 ${ }^{\mathrm{TM}}$ Real-Time System. Portiera and Hamiltonella were determined with the 16S rRNA gene, and the $\beta$-actin gene (nuclear gene) of whiteflies was measured in parallel for normalization. The relative density of the symbionts was defined as the ratio of bacterium single-copy genes to insect single-copy genes and calculated using the comparative CT method $\left(2^{-\Delta \Delta C t}\right)$. The primer sequences are listed in Supplementary Table 2.

\section{Statistical Analysis}

The data of developmental curves was analyzed via the Cox proportional-hazards model test, and other data of whitefly performance were analyzed using one-way analysis of variance (ANOVA), and Fisher's least significant difference (LSD) tests were used for ad hoc multiple comparisons. All the data analyses were performed using SPSS 20.0 Statistics.

\section{RESULTS}

\section{Diversity of Symbionts in Field Whitefly Populations}

We first analyzed genetic differentiation of the 17 field populations of MED whitefly based on $m t C O I$ gene sequence. All of the MED populations belong to the subclade Q1 (Figure 1A). Subsequently, we surveyed the diversity of symbionts in these MED populations. In addition to the primary symbiont Portiera, three secondary symbionts were found. Among them, Hamiltonella showed high infection frequency, $100 \%$ in 15 populations and 95 and $90.5 \%$ in the two remaining populations, respectively (Figure 1B and Supplementary Table 1). Moreover, phylogenetic analysis of the 16S rRNA sequence show that all of the Hamiltonella belong to the similar strains (Supplementary Figure 1). Cardinium was detected in 13 of the 17 whitefly populations with infection frequencies varying from 4.5 to 85.7\% (Figure 1B and Supplementary Table 1). Cardinium phylogenetic analyses indicated that the symbiont of $B$. tabaci were classified into four groups/strains (C1-C4) (Kanakala and Ghanim, 2019), and all of the tested Cardinum from the different populations clustered within the $\mathrm{C} 4$ group in the present study

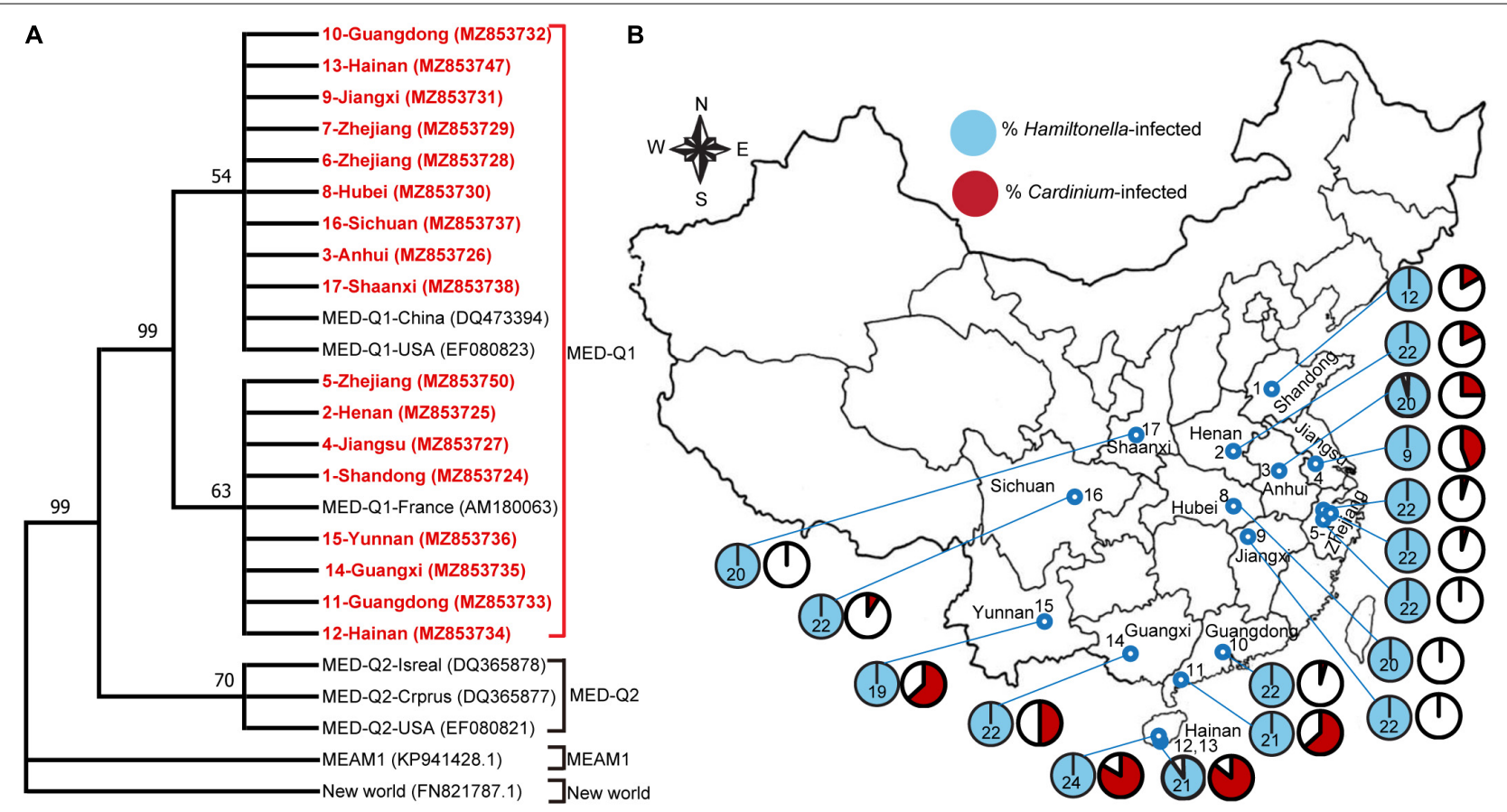

FIGURE 1 | The infection of secondary symbionts in field MED whitefly populations sampled in various locations in China. (A) Phylogenetic tree of the different whitefly populations based on the $m t C O /$ sequences ( 760 bp). Maximum Likelihood algorithms available in MEGA-X were used to infer phylogenetic relationships of the sequences that are shown as a cladogram. The $m t C O /$ sequences of $17 \mathrm{MED}$ populations from this study are indicated in bold red. The Genbank accession number is shown in bracket. Bootstrap values (>50\%) are shown on branches. (B) Infection frequencies of Cardinium and Hamiltonella in various geographical populations. The numbers in the map indicated the whitefly population No. The number in each of the pie charts indicates the sample size for PCR screens in that population. For details of the sample collection information, see Supplementary Table 1. 
(Supplementary Figure 2). Rickettsia was detected in only one of the 17 whitefly population with an infection frequency of $12.5 \%$ (Supplementary Table 1).

\section{Cardinium-Positive $\left(\mathrm{C}^{+}\right)$and Cardinium-Free $\left(\mathbf{C}^{-}\right)$Whitefly Lines}

We isolated Cardinium-positive $\left(\mathrm{C}^{+}\right)$and Cardinium-free $\left(\mathrm{C}^{-}\right)$ individuals from field population no. 11 of MED (Figure 1B and Supplementary Table 1) and introgressed to establish $\mathrm{C}^{+}$ and $\mathrm{C}^{-}$lines with a similar genetic background (Figure 2A). All individuals of the $\mathrm{C}^{+}$line harbored Portiera, Hamiltonella, and Cardinium, while those of the $\mathrm{C}^{-}$line harbored only Portiera and Hamiltonella (Figure 2B). Portiera and Hamiltonella were strictly located in the bacteriocytes in both lines of whiteflies (Figures 2C,D). While Cardinium were not restricted to any tissues or cells, with distribution in both the bacteriocytes and body cavity in the $\mathrm{C}^{+}$line (Figure 2E).

\section{Effects of Cardinium on Whitefly Fitness}

To investigate the effects of Cardinium on host fitness and reproduction, four treatments of mating were conducted between $\mathrm{C}^{+}$and $\mathrm{C}^{-}$lines including $\mathrm{C}^{+} \phi \times \mathrm{C}^{+} \sigma^{\top}, \mathrm{C}^{-} \phi \times \mathrm{C}^{-} \sigma^{\top}$, $\mathrm{C}^{+} \phi \times \mathrm{C}^{-} \sigma^{x}$, and $\mathrm{C}^{-} \phi \times \mathrm{C}^{+} \sigma^{x}$, and the fecundity and performance of progenies of the four mating treatments were
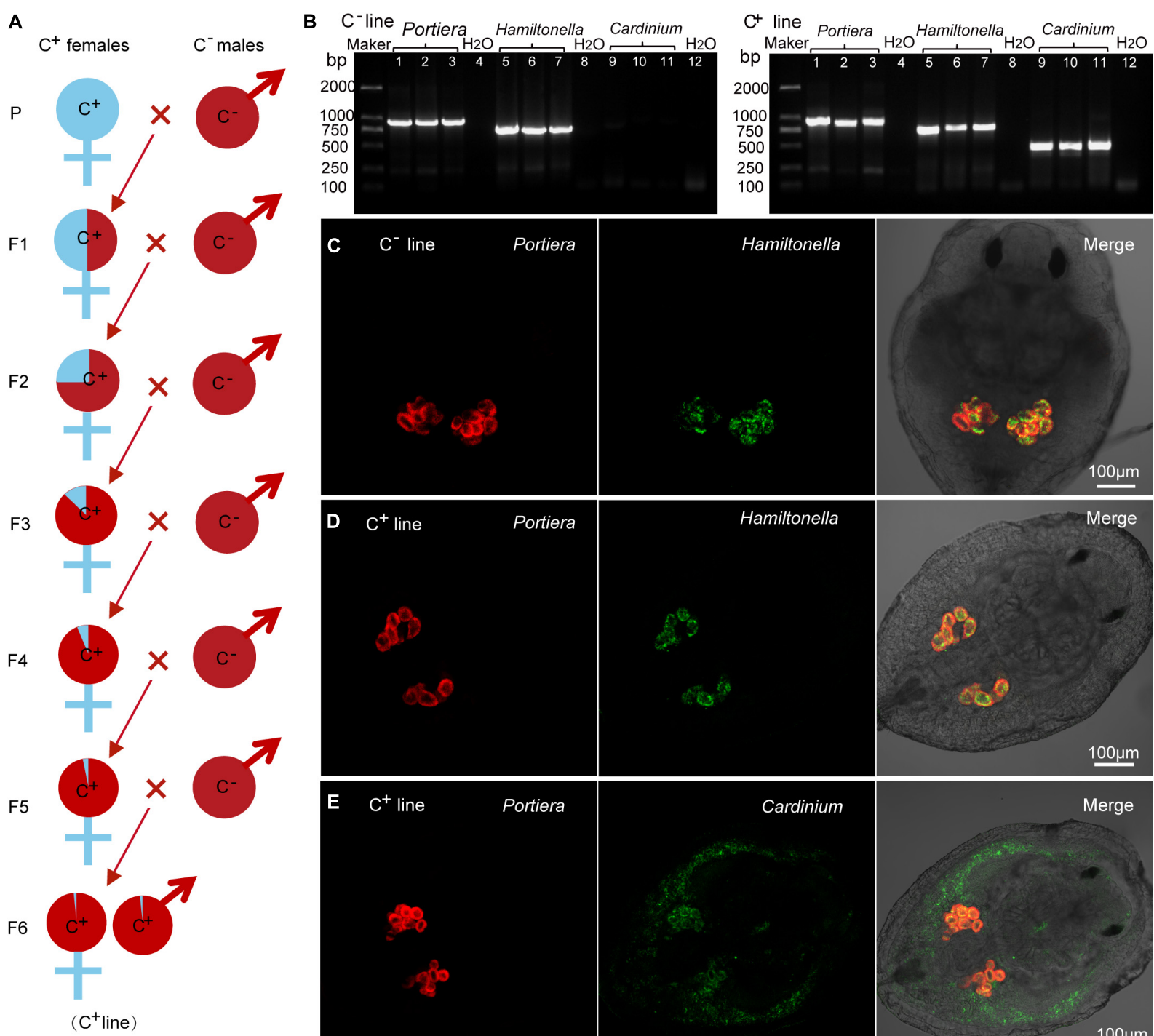

Hamiltonella
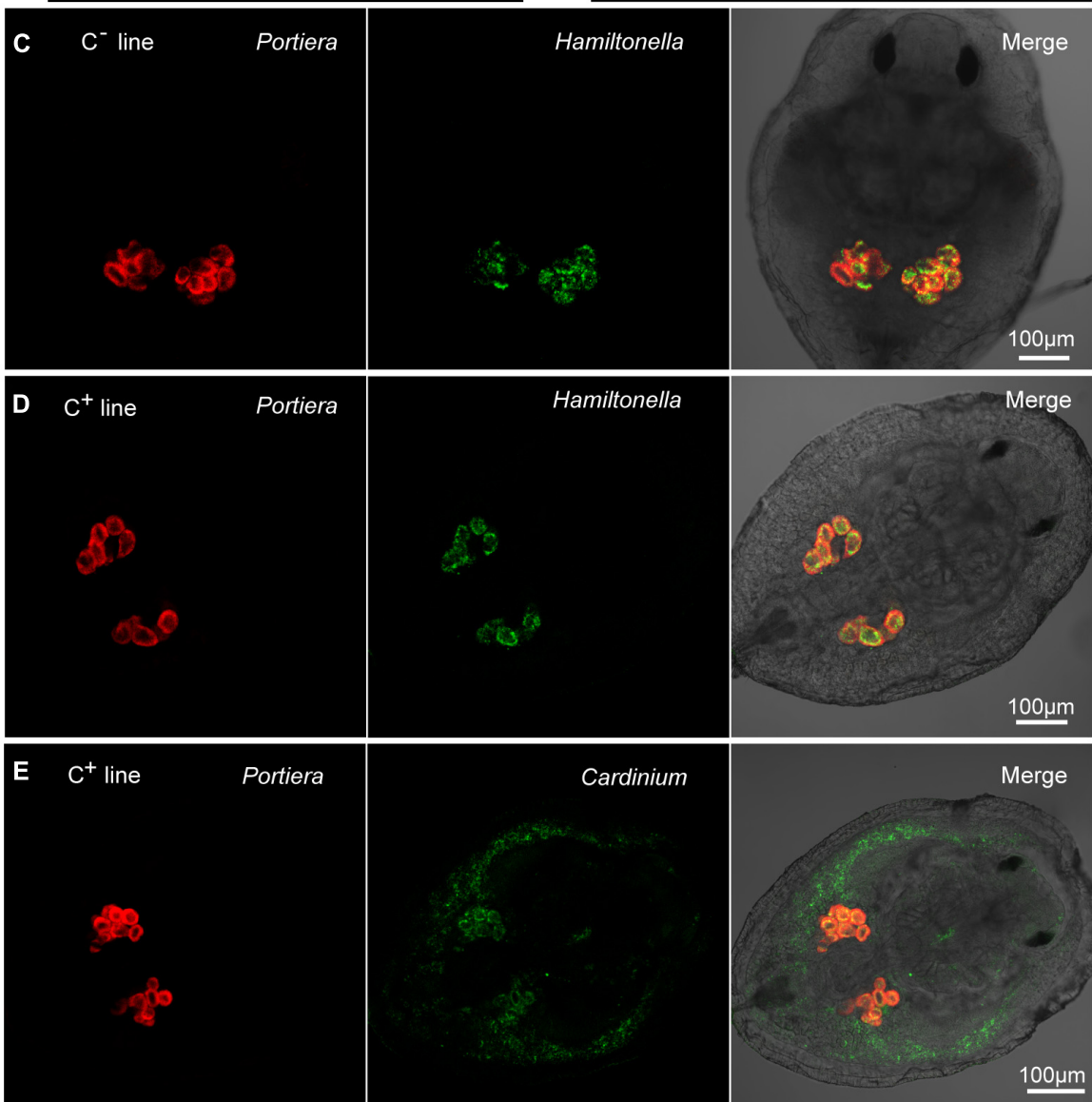

ge

FIGURE 2 | The presence/absence of symbionts in Cardinium-positive $\left(\mathrm{C}^{+}\right)$and Cardinium-free $\left(\mathrm{C}^{-}\right)$whitefly lines. (A) Establishment of $\mathrm{C}^{+}$and $\mathrm{C}^{-}$whitefly lines via introgression. The male $\mathrm{C}^{-}$whiteflies were introduced to mate with female $\mathrm{C}^{+}$whiteflies for 6 generations to homogenize their nuclear background. $\mathrm{P}$ : parental generation; F1-F6, the first to sixth filial generations. (B) Portiera, Hamiltonella and Cardinium were detected in $\mathrm{C}^{+}$line, and Portiera and Hamiltonella but not Cardinium were detected in $\mathrm{C}^{-}$line. Lane 1-3: Portiera; lane 5-7: Hamiltonella; lane 9-11: Cardinium; lane 4, 8, and 12: negative control (ddH $\left.\mathrm{H}_{2} \mathrm{O}\right)$. (C) The spatial distribution of Portiera (red) and Hamiltonella (green) in the $\mathrm{C}^{-}$line whitefly. (D) The spatial distribution of Portiera (red) and Hamiltonella (green) in the $\mathrm{C}^{+}$line whitefly. (E) The spatial distribution of Portiera (red) and Cardinium (green) in the $\mathrm{C}^{+}$line whitefly. 
assessed. The numbers of eggs produced by females in each of the two mating treatments $\mathrm{C}^{+}{ }_{+} \times \mathrm{C}^{+} \sigma^{7}$ and $\mathrm{C}^{+} q \times \mathrm{C}^{-} \sigma^{7}$ were significantly lower than females of $\mathrm{C}^{-} q \times \mathrm{C}^{-} \sigma^{-7}$ and $\mathrm{C}^{-} q \times \mathrm{C}^{+} \sigma^{-7}$ (Figure 3A; $\left.F_{(3,90)}=7.225, P<0.001\right)$. The hatchability of eggs of $\mathrm{C}^{+} \mathrm{O} \times \mathrm{C}^{+} \mathrm{O}^{-}$was lower than those of the remaining three mating treatments, while the survival rates from the 1st instar nymph to adulthood did not differ significantly among the four mating treatments (Figures 3B,C; $F_{(3,90)}=2.445, P=0.069$ for egg viability; $F_{(3,90)}=0.155, P=0.926$ for adult emergence). The development times from egg to adult emergence of the progenies of the two mating treatments $\mathrm{C}^{+} q \times \mathrm{C}^{+} \sigma^{7}$ and $\mathrm{C}^{+} q \times \mathrm{C}^{-} \sigma^{7}$ were longer than those of $\mathrm{C}^{-} q \times \mathrm{C}^{+} \sigma^{7}$ and $\mathrm{C}^{-} q \times \mathrm{C}^{-} \sigma^{\pi}$ (Figure 3D; $P=0.001)$. The numbers of progenies that reached adulthood of the two mating treatments $\mathrm{C}^{+}{ }_{+} \times \mathrm{C}^{+} \sigma^{7}$ and $\mathrm{C}^{+} q \times \mathrm{C}^{-} \sigma^{7}$ were lower than those of $\mathrm{C}^{-} q \times \mathrm{C}^{+} \sigma^{7}$ and $\mathrm{C}^{-} q \times \mathrm{C}^{+} \sigma^{7}$ (Figure 3E; $\left.F_{(3,90)}=4.645, P=0.005\right)$, while the sex ratios of the four mating treatments were similar (Figure 3F; $F_{(3,90)}=0.173$, $P=0.915)$.

Subsequently, we assessed the vertical transmission of Cardinium by investigating its infection in the progenies of the four treatments of mating between $\mathrm{C}^{+}$and $\mathrm{C}^{-}$ lines. In the two mating treatments $\mathrm{C}^{+}+\times \mathrm{C}^{+} \mathrm{O}^{x}$ and
$\mathrm{C}^{+} \mathrm{q} \times \mathrm{C}^{-} \mathrm{O}^{\top}$, where females were infected with Cardinium, nearly all individuals of their progenies were detected with Cardinium, while none of the progenies of $\mathrm{C}^{-}+\times \mathrm{C}^{+} \sigma^{\pi}$ and $\mathrm{C}^{-}+\times \mathrm{C}^{-} \sigma^{\top}$, where females were not infected with Cardinium, were detected with this bacterium (Supplementary Figure 3). These observations indicate that Cardinium is transmitted from female parent to progeny with high fidelity, and the infection status of parent male plays little or no role in the vertical transmission.

\section{Effects of Hamiltonella on Whitefly Reproduction}

To investigate the effects of Hamiltonella on the reproduction of MED whitefly, four treatments of mating between antibiotictreated and untreated F1 adult whiteflies were conducted, and the Hamiltonella status of F1 adults as well as the number and females $\%$ of F2 adult progenies were observed. In the F1 adults for mating experiments, the Hamiltonella were selectively depleted in both females and males using the antibiotic treatment (Figures 4A,B; $F_{(3,81)}=70.927, P<0.001$ for female; $F_{(3,81)}=15.296, P<0.001$ for male), while the abundance
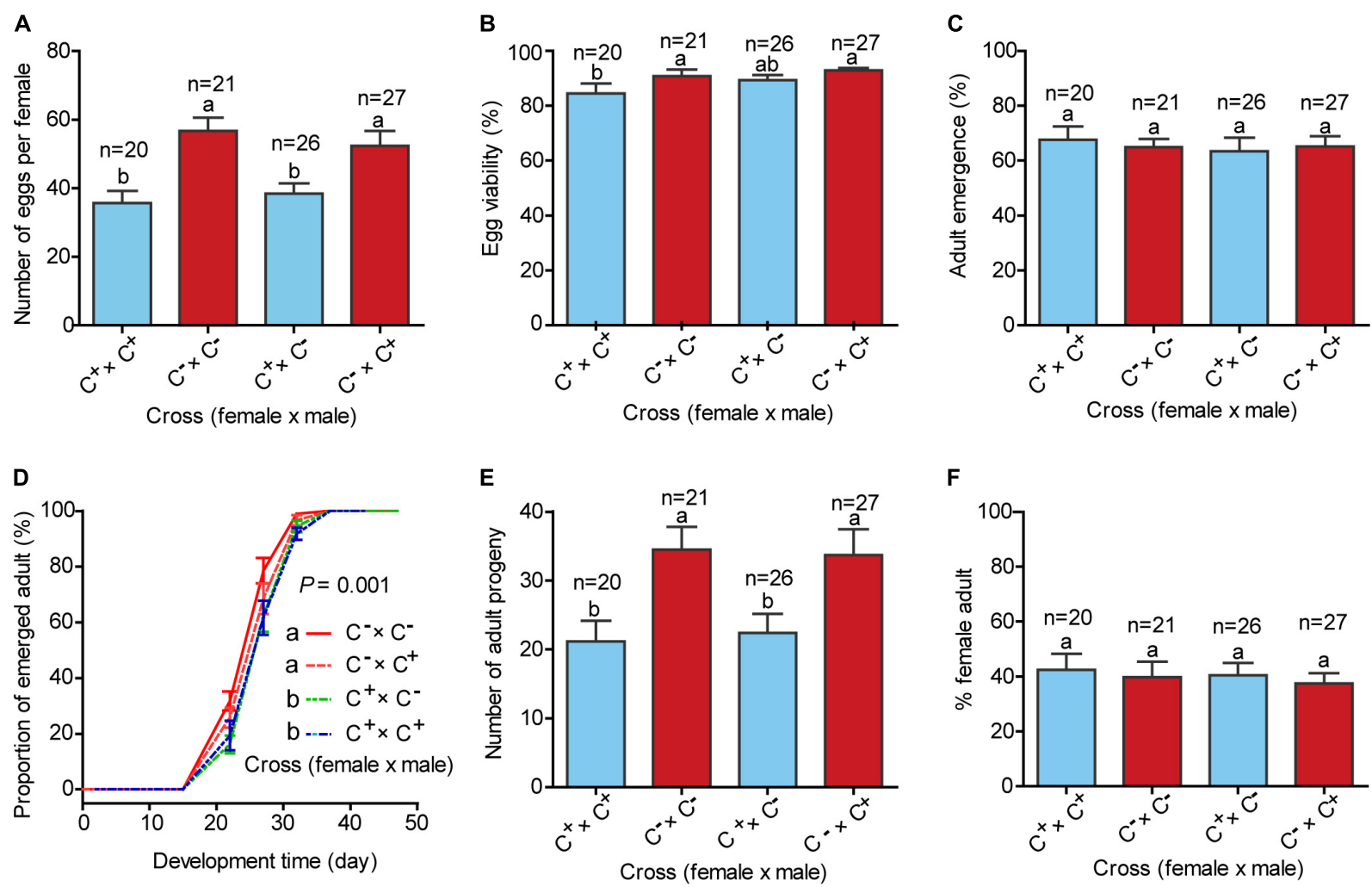

FIGURE 3 | Life history parameters of four treatments of mating between $\mathrm{C}^{+}$and $\mathrm{C}^{-}$whiteflies. (A) Mean number of eggs per female produced in 7 days; (B) Percentage of eggs that hatched; (C) Proportion of survival from the 1st instar nymph to adulthood; (D) Development time of progenies from egg to adulthood, expressed as the cumulative proportion of adult emergence; (E) Number of progenies that survived to adulthood; (F) Sex ratio of the adults. $\mathbf{C}^{+}$: the MED whitefly line harboring Portiera, Hamiltonelle and Cardinium; $\mathrm{C}^{-}$: the MED whitefly line harboring Portiera and Hamiltonelle but not Cardinium. In each replicate of the four treatments of mating, a female and a male were reared together to mate and oviposit for 7 days. Developmental curves were analyzed with the Cox proportional-hazards model tests, and other data were analyzed with one-way ANOVA followed by LSD test for multiple comparisons. The data are mean \pm SEM, and the different letters indicate significant differences at $P<0.05$. 


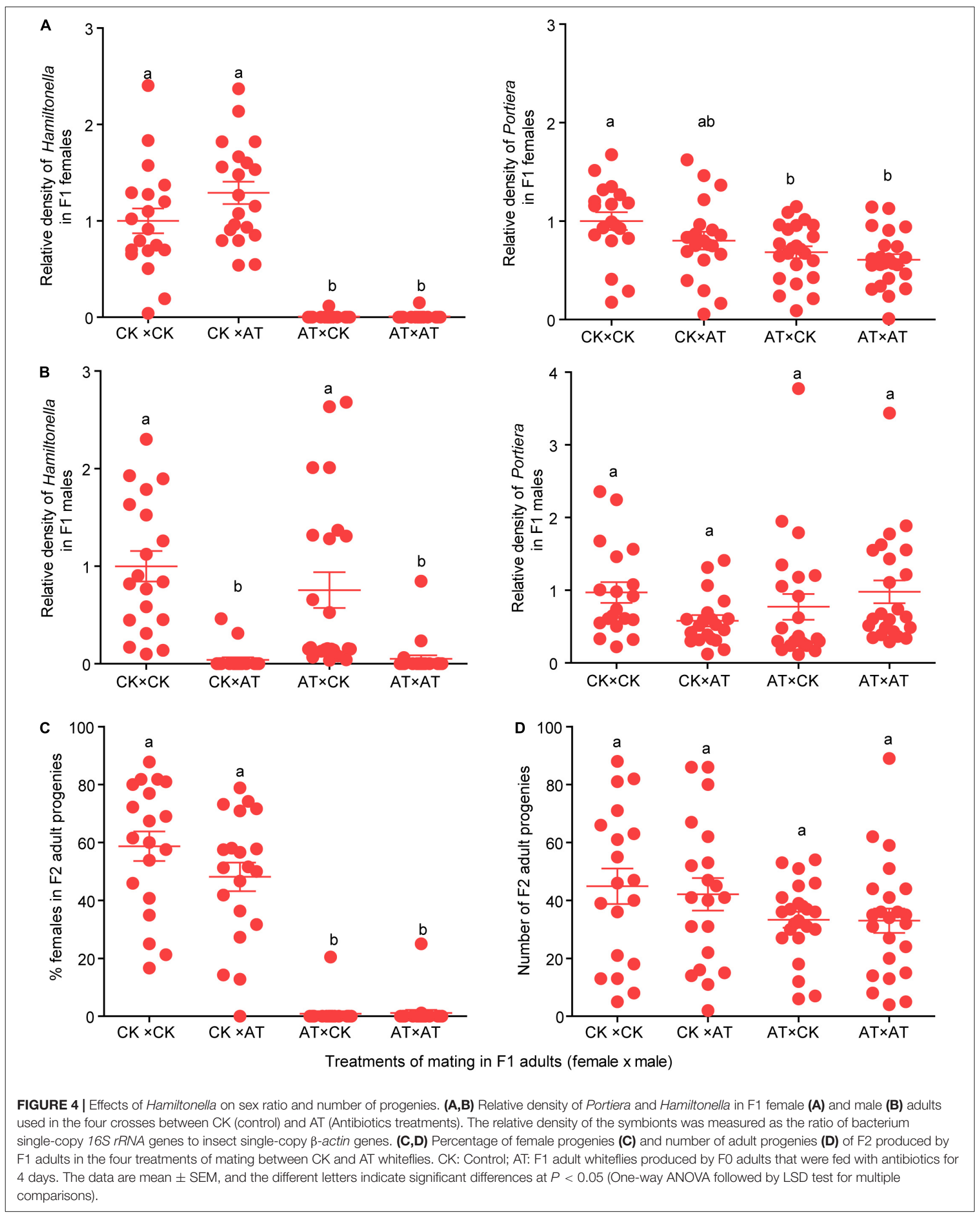


of Portiera was slightly reduced in females but not affected in males (Figures 4A,B; $F_{(3,81)}=5.136, P=0.003$ for female; $F_{(3,81)}=1.584, P=0.200$ for male). After the depletion of Hamiltonella, the female whiteflies produced extremely low percentage of female progenies, and the Hamiltonella status of the mating males had no significant effect on the sex ratio of the progenies (Figure 4C; $F_{(3,81)}=82.428, P<0.001$ ). However, two female whiteflies of F1 in the antibiotic treatment still harbored a low abundance of Hamiltonella (Figure 4A) and approximately $20 \%$ of their progenies were female (Figure 4C). In addition, the total number of progenies of Hamiltonella-free female whiteflies appeared slightly lower compared to that of untreated whiteflies (Figure 4D; $\left.F_{(3,81)}=1.669, P=0.180\right)$.

To determine the fidelity of vertical transmission of Hamiltonella in the host, effort was made to detect the bacterium in the progenies of the four treatments of mating between Hamiltonella-depleted whiteflies and non-antibiotic treated whiteflies. Hamiltonella was exclusively transmitted from the females to offspring, regardless of the Hamiltonella infection status of the mating males (Supplementary Figure 4).

\section{The Influence of Hamiltonella on Whitefly Fitness}

To assess other fitness costs/benefits of Hamiltonella on the whitefly host, we compared the performance of CK whiteflies (untreated and mated), unmated $\mathrm{CK}$ females (untreated and unmated), and AT whiteflies (Hamiltonella-depleted by antibiotic treatment). The three treatments did not differ significantly in egg viability, \% adult emergence, and number of progenies (Figures 5B,C,E; $F_{(2,55)}=0.406, P=0.668$ for egg viability; $F_{(2,55)}=0.253, P=0.777$ for adult emergence; $F_{(2,55)}=2.258$, $P=0.114$ for progeny size). The number of eggs produced per Hamiltonella-depleted female was significantly lower than that of CK females, but did not differ significantly from that of unmated CK females (Figure 5A; $F_{(2,55)}=2.306, P=0.109$ ). Similarly, the development rate of progenies produced by Hamiltonelladepleted females was slightly lower than that of mated CK females, but did not differ significantly from that of unmated CK females (Figure 5D; $P=0.266$ ). However, while $42 \%$ of the progenies produced by $\mathrm{CK}$ females were female, only $0.6 \%$ of the progenies produced by the Hamiltonella-depleted females were female, and none of the progenies produced by the unmated CK females was female as might be expected (Figure 5F; $\left.F_{(2,55)}=33.352, P<0.001\right)$.

\section{DISCUSSION}

The maternally inherited symbionts induced hosts to increase production of infected daughters by enhancing fitness or manipulating reproduction to facilitate the spread of symbiotic microorganisms in host populations. In this study, we surveyed the infection of two secondary symbionts Cardinium and
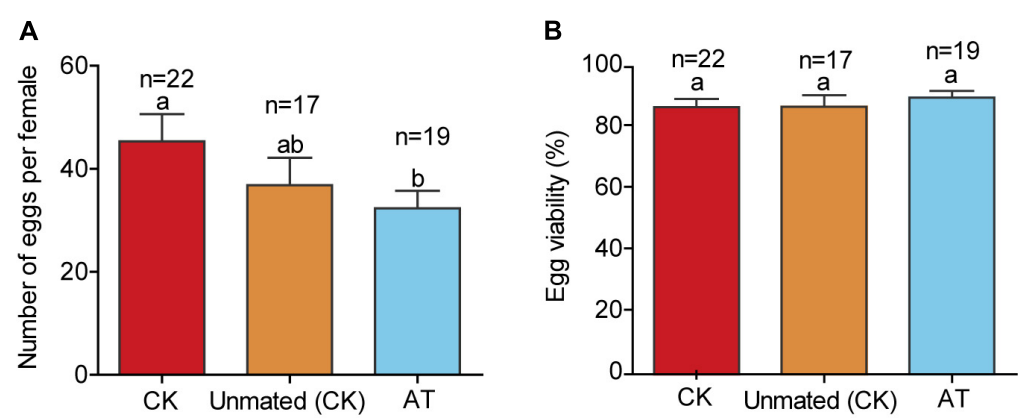

D

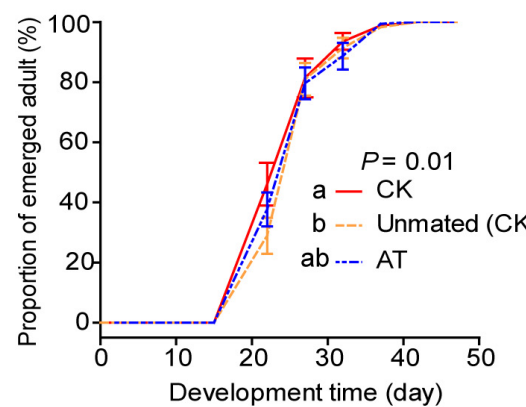

E

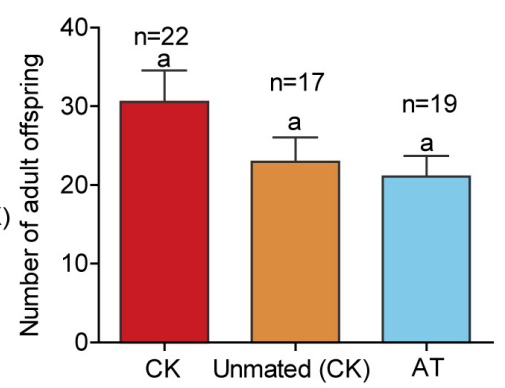

C

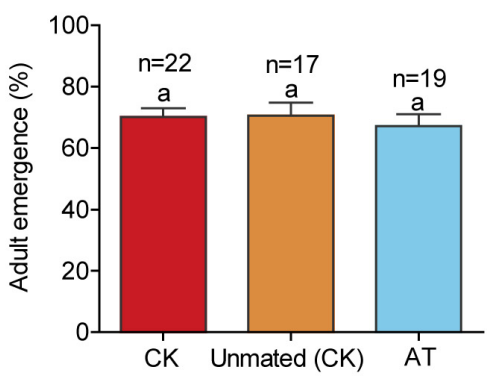

$\mathbf{F}$

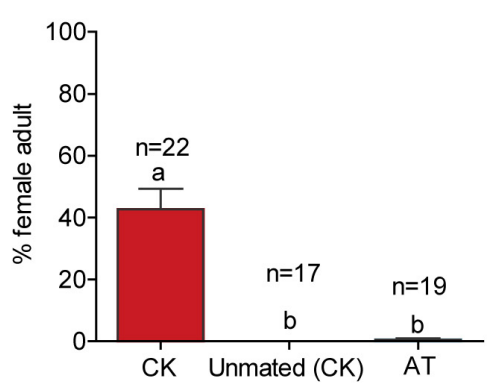

FIGURE 5 | Life history parameters of Hamiltonella-positive and Hamiltonella-free whiteflies on cotton plants. (A) Mean number of eggs per F1 female produced in 7 days; (B) Percentages of eggs that hatched; (C) Proportion of survival from the 1st instar nymph to adulthood; (D) Development time of progeny from egg to adulthood, expressed as the cumulative proportion of adult emergence; (E) The number of progenies that survived to adulthood; (F) Sex ratio of the adults. Unmated (CK): untreated virgin female adults; CK: untreated but mated females; AT: antibiotics-treated and mated females. In each replicate of Unmated CK, a female was allowed to oviposit for 7 days, and in each replicate of CK and AT, a female and a male were reared together to mate and oviposit for 7 days. The data are mean $\pm \mathrm{SEM}$, and the different letters indicate significant differences at $P<0.05$. 
Hamiltonella in field populations of the MED whitefly, and examined the effects of these two symbionts on the performance and reproduction of the whitefly host. Because the infection of Hamiltonella was essential to the host to survive normally through multiple generations, it was not feasible to create a MED whitefly line free of Hamiltonella but infected with Cardinium for the experiments. Nevertheless, the two MED whitefly lines used in the experiments for examining the functions of Cardinium differed only in the presence/absence of this symbiont, and the data obtained may be explored to understand the functions of the symbiont.

Our data show that Cardinium occurred widely in many field populations of the MED whitefly, but the infection frequencies were comparatively low and varied widely (Figure 1B and Supplementary Table 1). Cardinium is a widespread reproductive parasite and has been reported to induce parthenogenesis, cytoplasmic incompatibility and feminization in various arthropods (Weeks et al., 2001; ZchoriFein et al., 2001, 2004; Hunter et al., 2003). These reproductive manipulations skew reproduction to favor infected females specifically and then boost the spread of the symbiont in their insect hosts (Turelli and Hoffmann, 1991; Engelstädter and Telschow, 2009; Harris et al., 2010; Hurst and Frost, 2015). Our laboratory experiments show that, while Cardinium is strictly vertically transmitted in the MED whitefly (Supplementary Figure 3), its infection as well as mating with uninfected lines do not affect the host sex ratio of this haplodiploid insect (Figure 3F), indicating that this bacterium in the MED whiteflies does not act as a reproductive parasite. Therefore, reproductive manipulation is unlikely a major factor affecting the spread of Cardinium in field populations of its whitefly host, which is consistent with previous reports (Fang et al., 2014).

However, the infection of Cardinium incurred significant fitness costs on the MED whitefly, including reduction of fecundity and egg viability as well as delay in development of immature stages (Figure 3). Fecundity, egg viability, and development are biological features that often vary with host and symbiont genotypes as well as environmental conditions. There could be other fitness benefits/costs associated with the infection of Cardinium that were not examined in this study. For example, a recent study provides some preliminary indication indicates that Cardinium may increase the thermal tolerance of MED whitefly, which is likely associated with host genetic background (Yang et al., 2021). Considering the high temperature in southern China, thermal tolerance could be an important factor responsible for the high prevalence of Cardinium in some southern provinces, i.e., Yunnan, Guangxi, Guangdong, and Hainan (Figure 1B). In addition, in another symbiosis between parasitoid Encarsia inaron and Cardinium, while the Cardinium reduces initial fecundity, it also increases the host E. inaron longevity which may mitigate some of this fecundity cost (White et al., 2011). This, together with the negative and/or benefit association of these biological features under various conditions with the infection of Cardinium, seem to explain the comparatively low but widely varying infection frequencies of this symbiont in the field (Figure 1B and Supplementary Table 1).
The genus of Hamiltonella is mainly found in whiteflies and their related groups, including aphids and psyllids (Haynes et al., 2003; Russell et al., 2003). Hamiltonella confers resistance to parasitic wasps in some aphids (Oliver et al., 2003; Moran et al., 2005). However, in whiteflies, the Hamiltonella lost the virulent genes associated with this defensive function but contains genes that are involved in the production of essential nutrients in its genome (Rao et al., 2012; Rollat-Farnier et al., 2015). In whiteflies of the B. tabaci complex, Hamiltonella is mostly associated with the two widespread invasive species MEAM1 and MED in both their native regions of the Middle East and Mediterranean and regions of their invasion around the globe (Chiel et al., 2007; Gueguen et al., 2010; Bing et al., 2013a; Parrella et al., 2014; Zchori-Fein et al., 2014), and occasionally is found in some populations of the indigenous species New World 1 and New World 2 (De Marchi et al., 2018; Wang et al., 2019).

In the present study, the selective elimination of Hamiltonella from the MED whitefly resulted in strongly biased sex ratios, with an excess of male progenies (Figures 4A-C). This abnormal sex ratio has also been observed in the MEAM1 whitefly after Hamiltonella was experimentally depleted and was found to be associated with failure of egg fertilization rather than with failure of copulation (Shan et al., 2019), and in the geenhouse whitefly Trialeurodes vaporariorum after the removal of the symbiont Arsenophonus (Wang Y. B. et al., 2020). Both Hamiltonella and Arsenophonus contain genes that are capable of synthesizing B vitamins (Rollat-Farnier et al., 2015; Santos-Garcia et al., 2018). The removal of these symbionts reduced B vitamin levels and inhibited fertilization for the two whiteflies, and dietary B vitamin supplementation rescued fitness of the hosts (Wang Y. B. et al., 2020). In addition, the symbionts Wigglesworthia and Wolbachiaproduced B vitamins are significant for sexual maturation and reproduction in their diploid hosts tsetse flies and planthoppers, respectively (Snyder and Rio, 2015; Ju et al., 2020). In whiteflies, the $\mathrm{B}$ vitamin deficiency may influence the quality of eggs and sperm to prevent fertilization which results in a male-biased sex ratio in the haplodiploid insects. Thus, these symbionts are likely to provide the nutrients to benefit their whitefly host by influencing host sex allocation (Bondy and Hunter, 2019).

Relevant to these considerations is the impact of the maledominated sex ratio of Hamiltonella-free whiteflies on the prevalence of Hamiltonella in field whitefly populations. Where investigated, natural populations of $B$. tabaci MEAM1 and the subclade Q1 of $B$. tabaci MED have a high prevalence of this bacterium (Gueguen et al., 2010; Zchori-Fein et al., 2014), suggesting that Hamiltonella is transmitted vertically with high fidelity. However, failure of transmission occurs occasionally under adverse field conditions, especially high temperatures, because Hamiltonella has a greater sensitivity to high temperatures than its insect host (Wernegreen, 2012; Shan et al., 2014, 2017). Recovery of a symbiont is generally argued to be mediated by horizontal transmission, a process that occurs with exceptionally low frequency for Hamiltonella (Darby and Douglas, 2003; Oliver et al., 2014; Smith et al., 2015). However, a related possibility is that the production of male-dominated offspring by a Hamiltonella-free female whitefly would facilitate re-acquisition of Hamiltonella in natural populations 
with an intermediate prevalence of Hamiltonella. The greater the bias toward male offspring in a Hamiltonella-free female, the greater the probability that these males would mate with Hamiltonella-positive females yielding Hamiltonella-positive offspring. An alternative set of explanations for the prevalence of Hamiltonella is that the symbiont became incorporated into the host reproductive process over time, so that the loss of the symbiont is now detrimental to the host and may eventually cause the collapse of the host population due to overproduction of males. This outcome is in the selective interest of Hamiltonella, and also the insect if the host benefits from this interaction.

It is widely recognized that host genotype can greatly affect the benefits/costs conferred by a symbiont infection (Cass et al., 2016). Populations of $B$. tabaci MED consist of four phylogenetic clades. In theory, even small genetic variations within a particular clade of the host might still affect the benefits/costs associated with a symbiont infection. In addition, the symbiont Cardinium has been reported to consist of four subclades in $B$. tabaci (Kanakala and Ghanim, 2019). Thus, while the results of this study have some general implications in the ecology of the MED whitefly in China because Q1 is the predominant, widely distributed phylogenetic clade in this country, caution needs to be exerted when these implications are extended to understand the symbiotic association of the MED whitefly with Cardinium and Hamiltonella in other host populations and geographic regions.

Taken together, The data show that field populations of the B. tabaci MED whitefly in China are often coinfected with two secondary symbionts Cardinium and Hamiltonella, which differ in biological roles in the host. The beneficial and cost interactions of the co-infections with host are likely to play a critical role in determining the distribution of the symbionts in natural populations. Further research on the biological effects across different microbial symbionts would contribute to our understanding of the selective factors influencing the long-term maintenance and coevolution of multiple maternally inherited symbionts in whiteflies and other insects.

\section{DATA AVAILABILITY STATEMENT}

The original contributions presented in the study are included in the article/Supplementary Material, further inquiries can be directed to the corresponding author.

\section{REFERENCES}

Ayoubi, A., Talebi, A. A., Fathipour, Y., and Mehrabadi, M. (2020). Coinfection of the secondary symbionts, Hamiltonella defensa and Arsenophonus sp. contribute to the performance of the major aphid pest, Aphis gossypii (Hemiptera: aphididae). Insect Sci. 27, 86-98. doi: 10.1111/1744-7917.12603

Baumann, P. (2005). Biology of bacteriocyte-associated endosymbionts of plant sap-sucking insects. Annu. Rev. Microbiol. 59, 155-189. doi: 10.1146/annurev. micro.59.030804.121041

Bing, X. L., Ruan, Y. M., Rao, Q., Wang, X. W., and Liu, S. S. (2013a). Diversity of secondary endosymbionts among different putative species of the whitefly Bemisia tabaci. Insect Sci. 20, 194-206. doi: 10.1111/j.1744-7917.2012.01522.x

Bing, X. L., Yang, J., Zchori-Fein, E., Wang, X. W., and Liu, S. S. (2013b). Characterization of a newly discovered symbiont of the whitefly Bemisia tabaci

\section{AUTHOR CONTRIBUTIONS}

Both authors conceived and designed the study, wrote the manuscript, contributed to the article, and approved the submitted version. H-WS conducted the experimental work and data analysis.

\section{FUNDING}

This work was supported by The National Natural Science Foundation of China (Grant No. 31772173), the Natural Science Foundation of Zhejiang Province, China (Grant No. LQ21C140003), and the National Science Foundation for Postdoctoral Scientists of China (Grant No. 2020M681807).

\section{SUPPLEMENTARY MATERIAL}

The Supplementary Material for this article can be found online at: https://www.frontiersin.org/articles/10.3389/fmicb. 2021.739521/full\#supplementary-material

Supplementary Figure 1 | Phylogenetic position of Hamiltonella of different insect hosts based on the 16S rRNA sequences ( 720 bp). Maximum likelihood algorithms available in MEGA-X were used to infer phylogenetic relationships of the sequences that are shown as a cladogram. Hamiltonella sequences of 16 MED populations from this study are indicated in red. The Genbank accession number is shown in bracket. Bootstrap values (>50\%) are shown on branches.

Supplementary Figure 2 | Phylogenetic position of Cardinium of different whitefly populations based on the 16S rRNA sequences ( 390 bp). Maximum likelihood algorithms available in MEGA-X were used to infer phylogenetic relationships of the sequences that are shown as a cladogram. Cardinium sequences of 12 MED populations from this study are indicated in red. The Genbank accession number is shown in bracket. Bootstrap values (>50\%) are shown on branches.

Supplementary Figure $\mathbf{3}$ | Vertical transmission of Cardinium. (A) PCR detection of Cardinium in the progenies of four treatments of mating. (B) The transmission rates of Cardinium in the progenies of the four treatments of mating. The numbers above the columns indicate the positive total number of tested samples.

Supplementary Figure 4 | Vertical transmission of Hamiltonella. Relative density of Hamiltonella (A) and Portiera (B) in F2 progeny produced by F1 adults in the four treatments of mating between CK (control) and AT (Antibiotics treatments). The data are mean \pm SEM, and the different letters indicate significant differences at $P<0.05$ (One-way ANOVA followed by LSD test for multiple comparisons).

(Hemiptera: aleyrodidae). Appl. Environ. Micriobiol. 79, 569-575. doi: 10.1128/ AEM.03030-12

Bondy, E. C., and Hunter, M. S. (2019). "Sex ratios in the haplodiploid herbivores, Aleyrodidae and Thysanoptera: a review and tools for study," in Advances in Insect Physiology, ed. R. Jurenka (Cambridge: Academic Press), 251-281.

Bosco, D., Loria, A., Sartor, C., and Cenis, J. L. (2006). PCR-RFLP identification of Bemisia tabaci biotypes in the Mediterranean Basin. Phytoparasitica 34, 243-251.

Byrne, D. N., and Bellows, T. S. (1991). Whitefly biology. Annu. Rev. Entomol. 36, 431-457. doi: 10.1146/annurev.en.36.010191.002243

Cass, B. N., Himler, A. G., Bondy, E. C., Bergen, J. E., Fung, S. K., Kelly, S. E., et al. (2016). Conditional fitness benefits of the Rickettsia bacterial symbiont in an insect pest. Oecologia 180, 169-179. doi: 10.1007/s00442-0153436-x 
Chiel, E., Gottlieb, Y., Zchori-Fein, E., Mozes-Daube, N., Katzir, N., Inbar, M., et al. (2007). Biotype-dependent secondary symbiont communities in sympatric populations of Bemisia tabaci. Bull. Entomol. Res. 97, 407-413. doi: 10.1017/ S0007485307005159

Chu, D., Wan, F. H., Tao, Y. L., Liu, G. X., Fan, Z. X., and Bi, Y. P. (2008). Genetic differentiation of Bemisia tabaci (Gennadius) (Hemiptera: aleyrodidae) biotype Q based on mitochondrial DNA markers. Insect Sci. 15, 115-123. doi: 10.1111/j.1744-7917.2008.00191.x

Darby, A. C., and Douglas, A. E. (2003). Elucidation of the transmission patterns of an insect-borne bacterium. Appl. Environ. Microbiol. 69, 4403-4407. doi: 10.1128/aem.69.8.4403-4407.2003

De Barro, P. J., Liu, S. S., Boykin, L. M., and Dinsdale, A. B. (2011). Bemisia tabaci: a statement of species status. Annu. Rev. Entomol. 56, 1-19. doi: 10.1146/ annurev-ento-112408-085504

De Marchi, B. R., Kinene, T., Krause-Sakate, R., and Boykin, L. (2018). Comparative transcriptome analysis reveals genetic diversity in the endosymbiont Hamiltonella between native and exotic populations of Bemisia tabaci from Brazil. PLoS One 13:e0201411. doi: 10.1371/journal.pone.0201411

Douglas, A. E. (1989). Mycetocyte symbiosis in insects. Biol. Rev. 64, 409-434. doi: 10.1111/j.1469-185x.1989.tb00682.x

Douglas, A. E. (2015). Multiorganismal insects: diversity and function of resident microorganisms. Annu. Rev. Entomol. 60, 17-34. doi: 10.1146/annurev.ento. 43.1.17

Douglas, A. E., Francois, C. L. M. J., and Minto, L. B. (2006). Facultative 'secondary' bacterial symbionts and the nutrition of the pea aphid, Acyrthosiphon pisum. Physiol. Entomol. 31, 262-269. doi: 10.1111/j.1365-3032.2006.00516.x

Engelstädter, J., and Telschow, A. (2009). Cytoplasmic incompatibility and host population structure. Heredity 103, 196-207. doi: 10.1038/hdy.2009.53

Fang, Y. W., Liu, L. Y., Zhang, H. L., Jiang, D. F., and Chu, D. (2014). Competitive ability and fitness differences between two introduced populations of the invasive whitefly Bemisia tabaci Q in China. PLoS One 9:e100423. doi: 10.1371/ journal.pone.0100423

Feldhaar, H. (2011). Bacterial symbionts as mediators of ecologically important traits of insect hosts. Ecol. Entomol. 36, 533-543. doi: 10.1111/j.1365-2311.2011. 01318.x

Gauthier, N., Clouet, C., Perrakis, A., Kapantaidaki, D., Peterschmitt, M., and Tsagkarakou, A. (2014). Genetic structure of Bemisia tabaci MED populations from home-range countries, inferred by nuclear and cytoplasmic markers: impact on the distribution of the insecticide resistance genes. Pest Manag. Sci. 70, 1477-1491. doi: 10.1002/ps.3733

Ghosh, S., Bouvaine, S., Richardson, S. C. W., Ghanim, M., and Maruthi, M. N. (2018). Fitness costs associated with infections of secondary endosymbionts in the cassava whitefly species Bemisia tabaci. J. Pest Sci. 91, 17-28. doi: 10.1007/ s10340-017-0910-8

Gottlieb, Y., Ghanim, M., Chiel, E., Gerling, D., Portnoy, V., Steinberg, S., et al. (2006). Identification and localization of a Rickettsia sp. in Bemisia tabaci (Homoptera: aleyrodidae). Appl. Environ. Microbiol. 72, 3646-3652. doi: 10. 1128/AEM.72.5.3646-3652.2006

Gottlieb, Y., Ghanim, M., Gueguen, G., Kontsedalov, S., Vavre, F., Fleury, F., et al. (2008). Inherited intracellular ecosystem: symbiotic bacteria share bacteriocytes in whiteflies. FASEB J. 22, 2591-2599. doi: 10.1096/fj.07-101162

Gueguen, G., Vavre, F., Gnankine, O., Peterschmitt, M., Charif, D., Chiel, E., et al. (2010). Endosymbiont metacommunities, mtDNA diversity and the evolution of the Bemisia tabaci (Hemiptera: aleyrodidae) species complex. Mol. Ecol. 19, 4365-4378. doi: 10.1111/j.1365-294X.2010.04775.x

Harris, L. R., Kelly, S. E., Hunter, M. S., and Perlman, S. J. (2010). Population dynamics and rapid spread of Cardinium, a bacterial endosymbiont causing cytoplasmic incompatibility in Encarsia pergandiella (Hymenoptera: aphelinidae). Heredity 104, 239-246. doi: 10.1038/hdy. 2009.130

Haynes, S., Darby, A. C., Daniell, T. J., Webster, G., van Veen, F. J. F., Godfray, H. C. J., et al. (2003). Diversity of bacteria associated with natural aphid populations. Appl. Environ. Microbiol. 69, 7216-7223. doi: 10.1128/aem.69.12. 7216-7223.2003

Hendry, T. A., Hunter, M. S., and Baltrus, D. A. (2014). The facultative symbiont Rickettsia protects an invasive whitefly against entomopathogenic Pseudomonas syringae strains. Appl. Environ. Microbiol. 80, 7161-7168. doi: 10.1128/AEM. 02447-14
Himler, A. G., Adachi-Hagimori, T., Bergen, J. E., Kozuch, A., Kelly, S. E., Tabashnik, B. E., et al. (2011). Rapid spread of a bacterial symbiont in an invasive whitefly is driven by fitness benefits and female bias. Science 332, 254-256. doi: 10.1126/science.1199410

Hu, J., De Barro, P., Zhao, H., Wang, J., Nardi, F., and Liu, S. S. (2011). An extensive field survey combined with a phylogenetic analysis reveals rapid and widespread invasion of two alien whiteflies in China. PLoS One 6:e16061. doi: 10.1371/journal.pone.0016061

Hunter, M. S., Perlman, S. J., and Kelly, S. E. (2003). A bacterial symbiont in the Bacteroidetes induces cytoplasmic incompatibility in the parasitoid wasp Encarsia pergandiella. Proc. Biol. Sci. 270, 2185-2190. doi: 10.1098/rspb.2003. 2475

Hurst, G. D., and Frost, C. L. (2015). Reproductive parasitism: maternally inherited symbionts in a biparental world. Cold Spring Harb. Perspect. Biol. 7:a017699. doi: 10.1101/cshperspect.a017699

Ju, J. F., Bing, X. L., Zhao, D. S., Guo, Y., Xi, Z., Hoffmann, A. A., et al. (2020). Wolbachia supplement biotin and riboflavin to enhance reproduction in planthoppers. ISME J. 14, 676-687. doi: 10.1038/s41396-019-0559-9

Kanakala, S., and Ghanim, M. (2019). Global genetic diversity and geographical distribution of Bemisia tabaci and its bacterial endosymbionts. PLoS One 14:e0213946. doi: 10.1371/journal.pone.0213946

Lei, T., Zhao, J., Wamg, H. L., Liu, Y. Q., and Liu, S. S. (2021). Impact of a novel Rickettsia symbiont on the life history and virus transmission capacity of its host whitefly (Bemisia tabaci). Insect Sci. 28, 377-391. doi: 10.1111/1744-7917.12797

Liu, S. S., De Barro, P. J., Xu, J., Luan, J. B., Zang, L. S., Ruan, Y. M., et al. (2007). Asymmetric mating interactions drive widespread invasion and displacement in a whitefly. Science 318, 1769-1772. doi: 10.1126/science.1149887

Matalon, Y., Katzir, N., Gottlieb, Y., Portnoy, V., and Zchori-Fein, E. (2007). Cardinium in Plagiomerus diaspidis (Hymenoptera: encyrtidae). J. Invertebr. Pathol. 96, 106-108. doi: 10.1016/j.jip.2007.02.010

McLean, A. H. C., Parker, B. J., Hrcek, J., Kavanagh, J. C., Wellham, P. A. D., and Godfray, H. C. J. (2018). Consequences of symbiont co-infections for insect host phenotypes. J. Anim. Ecol. 87, 478-488. doi: 10.1111/1365-2656.12705

Misaka, B. C., Wosula, E. N., Marchelo-d'Ragga, P. W., Hvoslef-Eide, T., and Legg, J. P. (2020). Genetic diversity of Bemisia tabaci (Gennadius) (Hemiptera: aleyrodidae) colonizing sweet potato and cassava in South Sudan. Insects 11:58. doi: 10.3390/insects11010058

Moran, N. A., Degnan, P. H., Santos, S. R., Dunbar, H. E., and Ochman, H. (2005). The players in a mutualistic symbiosis: insects, bacteria, viruses, and virulence genes. Proc. Natl. Acad. Sci. U. S. A. 102, 16919-16926. doi: 10.1073/pnas. 0507029102

Nguyen, D. T., Morrow, J. L., Spooner-Hart, R. N., and Riegler, M. (2017). Independent cytoplasmic incompatibility induced by Cardinium and Wolbachia maintains endosymbiont coinfections in haplodiploid thrips populations. Evolution 71, 995-1008. doi: 10.1111/evo.13197

Oliver, K. M., and Higashi, C. H. (2019). Variations on a protective theme: Hamiltonella defensa infections in aphids variably impact parasitoid success. Curr. Opin. Insect Sci. 32, 1-7. doi: 10.1016/j.cois.2018.08.009

Oliver, K. M., Russell, J. A., Moran, N. A., and Hunter, M. S. (2003). Facultative bacterial symbionts in aphids confer resistance to parasitic wasps. Proc. Nat. Acad. Sci. U. S. A. 100, 1803-1807. doi: 10.1073/pnas.0335320100

Oliver, K. M., Smith, A. H., Russell, J. A., and Clay, K. (2014). Defensive symbiosis in the real world - advancing ecological studies of heritable, protective bacteria in aphids and beyond. Funct. Ecol. 28, 341-355. doi: 10.1111/1365-2435. 12133

Parrella, G., Nappo, A. G., Manco, E., Greco, B., and Giorgini, M. (2014). Invasion of the Q2 mitochondrial variant of Mediterranean Bemisia tabaci in southern Italy: possible role of bacterial endosymbionts. Pest Manag. Sci. 70, 1514-1523. doi: $10.1002 / \mathrm{ps} .3686$

Rao, Q., Wang, S., Su, Y. L., Bing, X. L., Liu, S. S., and Wang, X. W. (2012). Draft genome sequence of "Candidatus Hamiltonella defensa," an endosymbiont of the whitefly Bemisia tabaci. J. Bacteriol. 194:3558. doi: 10.1128/JB.00069-12

Rollat-Farnier, P. A., Santos-Garcia, D., Rao, Q., Sagot, M. F., Silva, F. J., Henri, H., et al. (2015). Two host clades, two bacterial arsenals: evolution through gene losses in facultative endosymbionts. Genome Biol. Evol. 7, 839-855. doi: 10.1093/gbe/evv030

Russell, J., Latorre, A., Sabater-Muñoz, B., Moya, A., and Moran, N. (2003). Side-stepping secondary symbionts: widespread horizontal transfer across and 
beyond the Aphidoidea. Mol. Ecol. 12, 1061-1075. doi: 10.1046/j.1365-294x. 2003.01780.x

Santos-Garcia, D., Juravel, K., Freilich, S., Zchori-Fein, E., Latorre, A., Moya, A., et al. (2018). To B or Not to B: comparative genomics suggests Arsenophonus as a source of B Vitamins in whiteflies. Front. Microbiol. 9:2254. doi: 10.3389/ fmicb.2018.02254

Shadmany, M., Boykin, L. M., Muhamad, R., and Omar, D. (2018). Genetic diversity of Bemisia tabaci (Hemiptera: aleyrodidae) species complex across Malaysia. J. Econ. Entomol. 112, 75-84. doi: 10.1093/jee/toy273

Shan, H. W., Deng, W. H., Luan, J. B., Zhang, M. J., Zhang, Z., Liu, S. S., et al. (2017). Thermal sensitivity of bacteriocytes constrains the persistence of intracellular bacteria in whitefly symbiosis under heat stress. Environ. Microbiol. Rep. 9, 706-716. doi: 10.1111/1758-2229.12554

Shan, H. W., Lu, Y. H., Bing, X. L., Liu, S. S., and Liu, Y. Q. (2014). Differential responses of the whitefly Bemisia tabaci symbionts to unfavorable low and high temperatures. Microb. Ecol. 68, 472-482. doi: 10.1007/s00248-014-0424-3

Shan, H. W., Luan, J. B., Liu, Y. Q., Douglas, A. E., and Liu, S. S. (2019). The inherited bacterial symbiont Hamiltonella influences the sex ratio of an insect host. Proc. Biol. Sci. 286:20191677. doi: 10.1098/rspb.2019.1677

Sloan, D. B., and Moran, N. A. (2012). Endosymbiotic bacteria as a source of carotenoids in whiteflies. Biol. Lett. 8, 986-989. doi: 10.1098/rsbl.2012.0664

Smith, A. H., Lukasik, P., O’Connor, M. P., Lee, A., Mayo, G., Drott, M. T., et al. (2015). Patterns, causes and consequences of defensive microbiome dynamics across multiple scales. Mol. Ecol. 24, 1135-1149. doi: 10.1111/mec.13095

Snyder, A. K., and Rio, R. V. (2015). “Wigglesworthia morsitans” folate (Vitamin B9) biosynthesis contributes to tsetse host fitness. Appl. Environ. Microbiol. 81, 5375-5386. doi: 10.1128/AEM.00553-15

Tsuchida, T., Koga, R., Horikawa, M., Tsunoda, T., Maoka, T., Matsumoto, S., et al. (2010). Symbiotic bacterium modifies aphid body color. Science 330, 1102-1104. doi: 10.1126/science. 1195463

Turelli, M., and Hoffmann, A. A. (1991). Rapid spread of an inherited incompatibility factor in California Drosophila. Nature 353, 440-442. doi: 10. 1038/353440a0

Wang, H. L., Lei, T., Xia, W. Q., Cameron, S. L., Liu, Y. Q., Zhang, Z., et al. (2019). Insight into the microbial world of Bemisia tabaci cryptic species complex and its relationships with its host. Sci. Rep. 9:6568. doi: 10.1038/s41598-01942793-8

Wang, R., Che, W., Wang, J., and Luo, C. (2020a). Monitoring insecticide resistance and diagnostics of resistance mechanisms in Bemisia tabaci Mediterranean (Q biotype) in China. Pestic. Biochem. Physiol. 163, 117-122. doi: 10.1016/j.pestbp. 2019.11.003

Wang, R., Wang, J., Zhang, J., Che, W., Feng, H., and Luo, C. (2020b). Characterization of flupyradifurone resistance in the whitefly Bemisia tabaci Mediterranean (Q biotype). Pest Manag. Sci. 76, 4286-4292. doi: 10.1002/ps. 5995

Wang, Y. B., Ren, F. R., Yao, Y. L., Sun, X., Walling, L. L., Li, N. N., et al. (2020). Intracellular symbionts drive sex ratio in the whitefly by facilitating fertilization and provisioning of B vitamins. ISME J. 14, 2923-2935. doi: 10.1038/s41396020-0717-0

Weeks, A. R., Marec, F., and Breeuwer, J. A. J. (2001). A mite species that consists entirely of haploid females. Science 292, 2479-2482. doi: 10.1126/ science. 1060411
Weeks, A. R., Velten, R., and Stouthamer, R. (2003). Incidence of a new sex-ratiodistorting endosymbiotic bacterium among arthropods. Proc. Biol. Sci. 270, 1857-1865. doi: 10.1098/rspb.2003.2425

Wernegreen, J. J. (2012). Mutualism meltdown in insects: bacteria constrain thermal adaptation. Curr. Opin. Microbiol. 15, 255-262. doi: 10.1016/j.mib. 2012.02.001

Werren, J. H., Baldo, L., and Clark, M. E. (2008). Wolbachia: master manipulators of invertebrate biology. Nat. Rev. Microbiol. 6, 741-751. doi: 10.1038/ nrmicro1969

White, J. A., Kelly, S. E., Cockburn, S. N., Perlman, S. J., and Hunter, M. S. (2011). Endosymbiont costs and benefits in a parasitoid infected with both Wolbachia and Cardinium. Heredity 106, 585-591. doi: 10.1038/hdy. 2010.89

Yang, K., Yuan, M. Y., Liu, Y., Guo, C. L., Liu, T. X., Zhang, Y. J., et al. (2021). First evidence for thermal tolerance benefits of the bacterial symbiont Cardinium in an invasive whitefly, Bemisia tabaci. Pest Manag. Sci. doi: 10.1002/ps.6543 [Epub Online ahead of Print].

Zchori-Fein, E., and Brown, J. (2002). Diversity of prokaryotes associated with Bemisia tabaci (Gennadius)(Hemiptera: aleyrodidae). Ann. Entomol. Soc. Am. 95, 711-718. doi: 10.1603/0013-87462002095[0711:DOPAWB]2.0.CO;2

Zchori-Fein, E., Gottlieb, Y., Kelly, S. E., Brown, J. K., Wilson, J. M., Karr, T. L., et al. (2001). A newly discovered bacterium associated with parthenogenesis and a change in host selection behavior in parasitoid wasps. Proc. Nat. Acad. Sci. U. S. A. 98, 12555-12560. doi: 10.1073/pnas.221467498

Zchori-Fein, E., Lahav, T., and Freilich, S. (2014). Variations in the identity and complexity of endosymbiont combinations in whitefly hosts. Front. Microbiol. 5:310. doi: 10.3389/fmicb.2014.00310

Zchori-Fein, E., Perlman, S. J., Kelly, S. E., Katzir, N., and Hunter, M. S. (2004). Characterization of a 'Bacteroidetes' symbiont in Encarsia wasps (Hymenoptera: aphelinidae): proposal of 'Candidatus Cardinium hertigii'. Int. J. Syst. Evol. Microbiol. 54, 961-968. doi: 10.1099/ijs.0.02957-0

Zhao, D., Hoffmann, A. A., Zhang, Z., Niu, H., and Guo, H. (2018). Interactions between facultative symbionts Hamiltonella and Cardinium in Bemisia tabaci (Hemiptera: aleyrodoidea): cooperation or conflict? J. Econ. Entomol. 111, 2660-2666. doi: 10.1093/jee/toy261

Conflict of Interest: The authors declare that the research was conducted in the absence of any commercial or financial relationships that could be construed as a potential conflict of interest.

Publisher's Note: All claims expressed in this article are solely those of the authors and do not necessarily represent those of their affiliated organizations, or those of the publisher, the editors and the reviewers. Any product that may be evaluated in this article, or claim that may be made by its manufacturer, is not guaranteed or endorsed by the publisher.

Copyright (c) 2021 Shan and Liu. This is an open-access article distributed under the terms of the Creative Commons Attribution License (CC BY). The use, distribution or reproduction in other forums is permitted, provided the original author(s) and the copyright owner(s) are credited and that the original publication in this journal is cited, in accordance with accepted academic practice. No use, distribution or reproduction is permitted which does not comply with these terms. 